\title{
The Structure of Aldehyde Dehydrogenase 16A1
}

Li-Kai Liu, and John J. Tanner*

Department of Biochemistry, University of Missouri, Columbia, Missouri

\begin{abstract}
ALDH16A1 is a unique and enigmatic member in the aldehyde dehydrogenase (ALDH) superfamily. Unlike other ALDHs, ALDH16A1 contains an additional Rossmann fold domain at the C-terminus of the protein. Furthermore, whereas other ALDHs utilizes nicotinamide adenine dinucleotide as a cofactor to catalyze myriad aldehyde substrates involved in aldehyde metabolism, mammalian ALDH16A1 lacks the universal active site cysteine, prompting speculation that the protein serves as a pseudo-enzyme that interacts with proteins such as maspardin, a membrane protein linked to neurological disorders. Also, a missense single nucleotide polymorphism in the $A L D H 16 A 1$ gene is a major risk factor in gout and serum uric acid levels. Curiously, ALDH16A1 from bacterial or lower animals contain the active cysteine, but whether these proteins have catalytic activity is unknown. Understanding the structure of ALDH16A1 is crucial to elucidate the roles of the protein in human health and diseases. We have targeted ALDH16A1 from human and several bacteria for crystal structure determination, analysis of the solution structural properties with SAXS and analytical ultracentrifugation, and enzyme activity measurments using a battery of aldehyde substrates. This presentation will report the latest results of this study, including the first crystal structure of ALDH16A1, determination of the oligomeric state in solution, and the demonstration that the bacterial enzymes exhibit ALDH catalytic activity, and determined the first structure of ALDH16A1.
\end{abstract}

